\title{
The Cancer Human Biobank
}

National Cancer Institute

\section{Source}

National Cancer Institute. The Cancer Human Biobank. NCI Thesaurus. Code C126485.

A derived program of the National Cancer Institute's Biorepositories and Biospecimen

Research Branch (NCI BBRB) that carries out specialized biospecimen and data

procurements to support biospecimen science activities. The caHUB network consists of Biospecimen Source Sites (BSSs), The Comprehensive Biospecimen Resource (CBR), The Comprehensive Data Resource (CDR), The Pathology Resource Center (PRC), and Molecular Analysis Facilities (MAFs). 